\title{
¿La metodología Flipped Classroom contribuye a crear un aprendizaje significativo?
}

\author{
Ana-Belén Escrig-Tena ${ }^{a}$, Mercedes Segarra-Ciprés ${ }^{b}$, Alexandra Badoiu ${ }^{c}$, Beatriz \\ García-Juan $^{\text {d }}$ \\ ${ }^{a}$ Universitat Jaume I, España, escrigt@uji.es, ${ }^{\text {b }}$ Universitat Jaume I, España, msegarra@uji.es, \\ ${ }^{\mathbf{c}}$ Universitat Jaume I, España, alexandra.badoiu@uji.es, ${ }^{\mathrm{d}}$ Universitat Jaume I, España, bjuan@uji.es
}

\section{Resumen}

En las últimas décadas se ha tratado de impulsar un cambio metodológico en la enseñanza universitaria desde una enseñanza centrada en la actividad del profesorado a otra orientada al aprendizaje del estudiantado. Así, en nuestra labor como docentes debemos ser capaces de seleccionar correctamente las acciones que emprendemos en el contexto de la asignatura, con la intención de que el estudiantado logre un aprendizaje profundo y comprensivo, y no tan sólo el procesamiento superficial de la información. Para ello es esencial fomentar actividades de aprendizaje que promuevan el papel activo del estudiantado, en las cuales "no se pueda escapar de aprender". En este sentido se han llevado a cabo experiencias en el aula teniendo en cuenta la denominada "clase al revés" (Flipped Classroom), la cual precisamente pretende fomentar el aprendizaje activo, colaborativo y la resolución de problemas. El propósito de este trabajo es valorar en qué grado los estudiantes perciben que han conseguido un aprendizaje significativo en el contexto de una asignatura en la que se ha seguido la metodología de la Flipped Classroom, así como valorar su satisfacción con la asignatura. Los resultados obtenidos proporcionan orientación al docente sobre la efectividad de las nuevas metodologías docentes en el proceso de aprendizaje.

Palabras clave: Flipped Classroom, aprendizaje significativo, satisfacción del estudiantado 


\section{Introducción}

El nuevo sistema de créditos ECTS promovido desde el Espacio Europeo de Educación Superior pretendía cambiar el modo de orientar la enseñanza, dirigiendo la mirada al estudiantado e impulsando el uso de nuevas metodologías docentes más enfocadas al desarrollo de competencias (Vidal, 2012). Así, el proceso de enseñanza-aprendizaje en este nuevo sistema debería estar enfocado en el aprendizaje del estudiantado más que en la docencia del profesorado.

Cruz Tomé (2002) define la enseñanza como el conjunto de decisiones, actividades y medios que se organizan sistemáticamente para facilitar el aprendizaje del alumno (la adquisición de conocimientos, habilidades, valores y actitudes), poniendo por tanto el énfasis en que la enseñanza debe realizarse de acuerdo con un método al servicio de un fin u objetivo que, en última instancia, es el aprendizaje. De acuerdo con Marqués et al. (2017), la docencia de cualquier asignatura debe proponer metodologías que promuevan un aprendizaje efectivo, y las sesiones meramente expositivas parecen no ser la mejor alternativa para lograrlo. La clase al revés o flipped classroom se presenta como una metodología docente alternativa a la clase magistral, que favorece la consecución de un aprendizaje significativo y al mismo tiempo contribuye a la satisfacción del estudiantado. A pesar del valor que puede tener esta metodología para mejorar el aprendizaje, como señala Prashar (2015), es necesario analizar la perspectiva del estudiantado en relación con el valor pedagógico que tiene la flipped classroom.

En este contexto, el presente trabajo trata de abordar la percepción del estudiantado sobre la flipped classroom. Más concretamente, el objetivo del estudio es analizar las opiniones del estudiantado, en una asignatura sobre gestión de la calidad en la que se ha utilizado la flipped classroom, en relación con el aprendizaje conseguido, el apoyo obtenido por el profesorado y sus compañeros, las exigencias de la asignatura, así como su satisfacción global con la misma.

\section{Marco conceptual}

De acuerdo con el marco conceptual que proporciona el constructivismo, el aprendiz es un procesador activo de la información y el profesorado es el facilitador de un aprendizaje significativo, el cual se concibe como un cambio estable en lo que sabe, hace y piensa el aprendiz. El término "aprendizaje significativo" fue acuñado por Ausubel (1982) a principios de los años 60, como oposición al aprendizaje repetitivo-memorístico, en el que 
no se relaciona, o se relaciona de forma arbitraria, lo que ha de ser aprendido con los conocimientos que el alumno posee. Esta relación inexistente o arbitraria hace que los conocimientos adquiridos de forma memorística no perduren.

Adoptamos, asimismo, el marco conceptual que ofrecen Biggs y Tang (2011) en su libro "Teaching for Quality Learning at University". Este enfoque parte de la premisa de que el aprendizaje tiene lugar a través del comportamiento activo del alumno. Citando a Tyler (1949) "Learning takes place through the active behavior of the student: it is what he does that he learns, not what the teacher does". Biggs y Tang (2011) proponen siete características que debe poseer un buen entorno de aprendizaje, las cuales son resumidas por Marqués et al. (2017) según se presenta en la Tabla 1.

Esta concepción del proceso de enseñanza-aprendizaje convierte la ocupación del docente en una tarea compleja y retadora, puesto que en el desempeño de su actividad debe seleccionar correctamente las acciones que emprende con la intención de que el estudiantado logre un aprendizaje profundo y comprensivo, y no tan sólo el procesamiento superficial de la información (Lloret y Mir, 2007).

Tabla 1. Características de un buen entorno de aprendizaje para la adquisición de competencias.

\begin{tabular}{|l|l|}
\hline \multicolumn{1}{|c|}{ Característica } & \multicolumn{1}{c|}{ Definición } \\
\hline $\begin{array}{l}\text { Aprendizaje } \\
\text { reflexivo }\end{array}$ & $\begin{array}{l}\text { Un buen entorno de aprendizaje debe dar al alumno oportunidades para reflexionar: } \\
\text { ¿cómo me va? iestoy cometiendo errores? ihay algún patrón en mis errores? icómo } \\
\text { puedo evitar dichos errores? ¿hay alguna forma mejor de hacerlo? }\end{array}$ \\
\hline Estar activos & $\begin{array}{l}\text { Aprendemos activando los sentidos y cuantos más sentidos se activan, más efectivo es el } \\
\text { aprendizaje ya que los sentidos se refuerzan. Si el aprendizaje se hace haciendo tareas, } \\
\text { aunque sea un aprendizaje de tipo declarativo, es más fácil recordar lo aprendido. }\end{array}$ \\
\hline $\begin{array}{l}\text { Feedback } \\
\text { formativo }\end{array}$ & $\begin{array}{l}\text { Se proporciona durante el proceso de aprendizaje y le sirve al alumno para saber cómo } \\
\text { va y qué ha de hacer para lograr los resultados de aprendizaje. Se trata de utilizar los } \\
\text { posibles errores cometidos de forma constructiva. Es necesario crear un clima en el que } \\
\text { el alumnado no se sienta juzgado. }\end{array}$ \\
\hline Motivación & $\begin{array}{l}\text { Según la teoría valor-expectativa, hay dos factores que hacen que alguien quiera } \\
\text { aprender algo: tiene que ser importante, debe tener valor para el aprendiz (debe estar } \\
\text { relacionado con los resultados de aprendizaje), y el aprendiz necesita sentir que va a } \\
\text { conseguirlo, que tendrá éxito (para lo cual es importante conocer los criterios mediante } \\
\text { los cuales saber si han tenido éxito). }\end{array}$ \\
\hline $\begin{array}{l}\text { Conocimiento } \\
\text { base } \\
\text { interconectado }\end{array}$ & $\begin{array}{l}\text { Según la teoría del constructivismo, al aprender se reestructura lo que se sabe para } \\
\text { conectarlo con lo nuevo. Para facilitar que los alumnos hagan dichas conexiones, el } \\
\text { profesorado debe construir sobre lo conocido haciendo conexiones de manera explícita, } \\
\text { poniendo ejemplos que resulten familiares, pidiendo a los alumnos que expliquen sus } \\
\text { experiencias, haciendo paralelismos, conectando con otras materias, temas, etc. }\end{array}$ \\
\hline $\begin{array}{l}\text { Eprendizaje } \\
\text { calidad }\end{array}$ & $\begin{array}{l}\text { Exandamental promover situaciones donde los alumnos aprendan unos de otros. } \\
\text { ven las cosas de otra manera. }\end{array}$ \\
\hline $\begin{array}{l}\text { Hay que usar variedad de métodos. Las sesiones de clase han de estar bien estructuradas } \\
\text { y deben seguir un ritmo adecuado. }\end{array}$ \\
\hline
\end{tabular}

Fuente: adaptado de Biggs y Tang (2011) y Marqués et al. (2017). 
De acuerdo con Biggs y Tang (2011), las actividades deben ser diseñadas de modo que el estudiante no pueda pasar por ellas sin aprender. Más concretamente, Bain (2006) concluye que los mejores profesores son los que preparan sus clases a partir de lo que quieren que sus estudiantes hagan y plantea 4 preguntas que los buenos profesores se cuestionan: “¿qué deberían hacer intelectual, física y/o emocionalmente mis alumnos?, ¿cómo puedo ayudarles? ¿cómo podemos mis alumnos y yo entender mejor el aprendizaje efectuado? ¿cómo puedo evaluar mis intentos de fomentar ese aprendizaje?

Por su parte el profesor Finkel (2008), en su libro "Dar clase con la boca cerrada", inspirado en la máxima de que "ninguna idea puede transmitirse de una persona a otra" (Dewey, 1916), propone la utilización de métodos que puedan complementar o sustituir la pura transmisión oral de conocimientos y técnicas, y fomentar el papel activo del estudiantado, partiendo del supuesto de que lo aprendido tiene valor si nos enfrentamos a problemas e intentamos resolverlos. Este autor propone la lectura de textos relevantes seleccionados por el profesorado, invitar a la discusión o promover la indagación como método de aprendizaje a través, por ejemplo, de talleres conceptuales.

A partir de estos referentes, la clase al revés o flipped classroom se erige como una metodología que puede fomentar el aprendizaje significativo. La clase al revés se concibe como una alternativa a las sesiones expositivas, y pretende fomentar el aprendizaje activo, colaborativo y la resolución de problemas (Abeysekera y Dawson, 2015). La metodología docente basada en la flipped classroom consiste en que el alumnado trabaja contenidos antes de la sesión de clase, de forma no presencial, y una vez en el aula se proponen actividades de aprendizaje para trabajar niveles cognitivos más altos de acuerdo con la taxonomía de Bloom (p.e. analizar, aplicar, evaluar, crear) (Marqués et al., 2017). Antes de la clase se proporcionan materiales en forma de artículos de prensa o académicos, vídeos, y se les proponen actividades previas para trabajarlos. Además, es habitual que estas actividades previas se apoyen en la presencia de las nuevas tecnologías. En la sesión del aula se realizan actividades de aprendizaje para profundizar, se detectan y analizan errores, se aclaran dudas, etc. De este modo, se convierte el tiempo de clase presencial en un espacio en que los alumnos están activos trabajando juntos y con el apoyo del profesorado. En este contexto, la labor del profesorado pasa de la elaboración de presentaciones a la preparación de actividades para que el estudiantado trabaje tanto previamente a la sesión de clase como en la sesión presencial en el aula, de modo que se pueda profundizar en el aprendizaje y éste pueda ser retenido (Roehl et al., 2013).

De acuerdo con Perdomo (2016), en esta metodología, la autorreflexión del estudiantado a partir de las actividades previas a la sesión de clase es clave y le permitirá hacerse las preguntas oportunas para que en la sesión de clase pueda aprovechar las actividades propuestas y el profesorado pueda guiarle en su proceso de aprendizaje. En la misma línea argumental, Prashar (2015) señala que la flipped classroom favorece que el alumnado 
"pruebe" o constate por él mismo el contenido a aprender, así como que se sientan más abiertos a la asociación de contenidos y materias. De este modo, la flipped classroom puede contribuir a un aprendizaje significativo por parte del estudiantado al permitirle construir nuevo conocimiento y habilidades que tienen un sentido a partir de sus conocimientos previos, y al incrementar, al mismo tiempo, su percepción de responsabilidad para con su aprendizaje debido al protagonismo que se les otorga (Barreiro y Novo, 2018). Así lo confirman los estudios realizados por varios profesores universitarios de diferentes ámbitos y territorios (e.g. Asef-Vaziri, 2015; Prashar, 2015; Perdomo et al., 2016; Esteban, 2018).

En definitiva, las principales ventajas que se desprenden de esta metodología pueden resumirse en que: el alumnado puede progresar en su casa a su propio ritmo, el tiempo en el aula es invertido de un modo más creativo y efectivo, el estudiantado se involucra en mayor grado en su propio proceso de aprendizaje, el docente obtiene una mejor comprensión de las dificultades y los diferentes estilos de aprendizaje del estudiantado al hacer las actividades de clase y, por último, aunque no menos importante, al estudiantado le gusta y disfruta con esta metodología (Fulton, 2012; Herreid y Schiller, 2013).

\section{Metodología}

Con el propósito de conocer la percepción del estudiantado sobre la flipped classroom, en el curso 2017-18 se realizó una encuesta al alumnado matriculado en la asignatura Gestión de la calidad y recursos humanos, impartida en el cuarto curso del Grado en Relaciones Laborales y Recursos Humanos de la Universitat Jaume I, en Castellón (España). Esta asignatura se planificó de manera que el alumnado tenía que realizar una serie de actividades previas a las diferentes sesiones de clase para trabajar algunos de los resultados de aprendizaje. Por ejemplo, en la actividad que se trabaja el concepto de calidad, los alumnos debían visualizar dos vídeos cortos y leer una nota técnica, a partir de los cuales debían responder a unas preguntas, incluidas en un formulario de Google, sobre los diferentes enfoques para comprender el concepto de calidad. Asimismo, en el formulario podían plantear las dudas que les habían surgido, para poder comentarlas en clase. Con posterioridad, en la sesión de clase, se comentan las dudas y se profundiza con el desarrollo de otras actividades realizadas en equipo y con la tutorización de la profesora. Se consiguió una muestra de 28 alumnos, sobre los 30 matriculados, lo cual nos permite contar con una muestra representativa.

El cuestionario incluía una cuestión para que el estudiantado valorara si el aprendizaje conseguido en la clase al revés había sido mejor, igual o peor que el obtenido en el caso de 
la clase expositiva. Asimismo, para valorar el aprendizaje significativo se incluyeron 6 ítems elaborados a partir de la escala utilizada por Guirao et al. (2007). Dado que la clase al revés requiere la realización de actividades en el aula que requieren el apoyo del profesorado y de los compañeros, se incluyeron cuestiones dirigidas a valorar el apoyo por parte de la profesora así como de sus compañeros, o sobre la carga de trabajo percibida, los cuales se seleccionaron a partir de investigaciones como las de van Yperen y Hagedoorn (2003) y van Wanrooy et al. (2013). Finalmente, se incluyó un ítem para valorar la satisfacción general con la asignatura, y una variable de clasificación sobre la asistencia regular a las sesiones presenciales. En todos los ítems en los que se pedía la valoración del estudiantado se utilizó una escala Likert de 10 puntos.

Para el análisis de los datos se ha utilizado el programa estadístico SPSS y, en concreto, se ha realizado un análisis descriptivo de medias, desviaciones típicas y análisis de frecuencias, así como análisis de correlaciones.

\section{Resultados}

El $89 \%$ del estudiantado percibe que la realización de las actividades con antelación a la clase, les ha permitido aprender mejor que si se hubiese seguido la metodología de la clase magistral. Únicamente un $11 \%$ considera que igual, y ningún alumno considera que peor. Algunas opiniones sobre la clase al revés manifestadas en el cuestionario afirman que "se aprende mejor porque desarrollas conocimientos por ti mismo, y luego los refuerzas en clase", "pienso que ayuda a entender mejor la materia y es una forma de motivar ya que dichas actividades puntúan" o "toda enseñanza que requiera el proceso de pensar por uno mismo es positiva, en lugar de escuchar o creer todo lo que se nos dice sin cuestionar nada".

En relación con el aprendizaje significativo, la tabla 2 muestra las valoraciones medias referidas a los 6 ítems recogidos en el cuestionario, así como el promedio de los mismos.

Tabla 2. Aprendizaje significativo $(\mathrm{N}=28)$

\begin{tabular}{|l|c|c|c|c|c|}
\hline & Media & Mediana & Desv. típ. & $\begin{array}{c}\text { \% punt. } \\
\mathbf{1 - 3}\end{array}$ & $\begin{array}{c}\text { \% punt. } \\
\mathbf{8 - 1 0}\end{array}$ \\
\hline $\begin{array}{l}\text { 1. La metodología utilizada me ha permitido ir } \\
\text { desarrollando mi aprendizaje de forma paulatina }\end{array}$ & 8.50 & 9.00 & 1.774 & 3.6 & 85.7 \\
\hline $\begin{array}{l}\text { 2. La profesora consideró mi conocimiento previo y sus } \\
\text { observaciones me ayudaron a mejorar mi conocimiento }\end{array}$ & 8.28 & 8.00 & 1.429 & 0 & 72 \\
\hline $\begin{array}{l}\text { 3. Los contenidos trabajados de forma autónoma me han } \\
\text { permitido desarrollar mi conocimiento }\end{array}$ & 8.74 & 9.00 & 1.318 & 0 & 85.2 \\
\hline $\begin{array}{l}\text { 4. El nuevo conocimiento presentado se conectó con el } \\
\text { conocimiento previo que tenía }\end{array}$ & 7.23 & 7.50 & 2.215 & 7.7 & 50 \\
\hline $\begin{array}{l}\text { 5. La metodología de aprendizaje utilizada me permite } \\
\text { conseguir los objetivos planteados en la materia }\end{array}$ & 8.59 & 9.00 & 1.421 & 0 & 81.5 \\
\hline $\begin{array}{l}\text { 6. El aprendizaje que voy adquiriendo es más perdurable } \\
\text { que el aprendizaje memorístico }\end{array}$ & 8.96 & 9.00 & 1.105 & 0 & 89.3 \\
\hline Media aprendizaje significativo & 8.38 & 8.33 & 1.01 & 0 & 64.3 \\
\hline
\end{tabular}


Como se observa en la tabla 2, la percepción de la metodología flipped classroom como medio para desarrollar el aprendizaje de forma paulatina presenta una media alta, recibiendo un $85.7 \%$ de las respuestas puntuaciones entre 8 y 10 . El aprendizaje adquirido es considerado más perdurable que el aprendizaje memorístico, siendo este el ítem que presenta la valoración más alta (8.96). Por otra parte, la media más baja corresponde a la percepción de los estudiantes sobre la conexión del nuevo conocimiento con el conocimiento previo que se tenía. Se observa que el porcentaje de las puntuaciones entre 1 y 3 son inferiores al $7.7 \%$ en todos los ítems del aprendizaje significativo.

Respecto a la percepción sobre el apoyo por parte de la profesora y de los compañeros y la carga de trabajo que perciben en la asignatura, la tabla 3 muestra que el alumnado percibe un elevado apoyo por parte de la profesora (valoración media de 9.47), destacando el trato justo percibido o el cumplimiento de las promesas por parte de la profesora, ítems en el que el $100 \%$ de los encuestados sitúan su valoración por encima de 8. En cuanto al apoyo de los compañeros, igual que al apoyo de la profesora, ningún estudiante ha puntuado por debajo de 3 su respuesta, y la valoración media (8.23) se puede considerar alta, aunque inferior al apoyo percibido por la profesora. Por otra parte, el estudiantado no percibe que exista una elevada carga de trabajo en la asignatura (media de 5.05).

Tabla 3. Apoyo y carga percibidas por los estudiantes $(\mathrm{N}=\mathbf{2 8})$

\begin{tabular}{|c|c|c|c|c|c|}
\hline & Media & Mediana & $\begin{array}{l}\text { Desv. } \\
\text { típ. }\end{array}$ & \% punt. & \% punt. \\
\hline \multicolumn{6}{|l|}{ APOYO PROFESORA } \\
\hline 1. Se puede confiar en que cumplirá sus promesas & 9.61 & 10.00 & 0.629 & 0 & 100 \\
\hline $\begin{array}{l}\text { 2. Es sincera al intentar entender el punto de vista de } \\
\text { los estudiantes }\end{array}$ & 9.39 & 10.00 & 1.031 & 0 & 92.9 \\
\hline 3. Tratan a los estudiantes honestamente & 9.57 & 10.00 & 0.836 & 0 & 92.9 \\
\hline $\begin{array}{l}\text { 4. Entiende que los estudiantes tengan que atender } \\
\text { otras tareas además de las de la asignatura }\end{array}$ & 9.07 & 10.00 & 1.412 & 0 & 88.09 \\
\hline 5. Trata a los estudiantes justamente & 9.71 & 10.00 & 0.535 & 0 & 100 \\
\hline Media apoyo profesora & 9.47 & 9.60 & 0.73 & 0 & 92.9 \\
\hline \multicolumn{6}{|l|}{ APOYO COMPAÑEROS } \\
\hline $\begin{array}{l}\text { 1. Puedo confiar en mis compañeros/as cuando las } \\
\text { cosas se ponen difíciles }\end{array}$ & 7.78 & 8.00 & 1.968 & 0 & 70,4 \\
\hline $\begin{array}{l}\text { 2. Si es necesario, puedo pedir ayuda a mis } \\
\text { compañeros/as }\end{array}$ & 8.64 & 9.00 & 1.471 & 0 & 75.00 \\
\hline Media apoyo compañeros & 8.23 & 9.00 & 1.63 & 0 & 71.4 \\
\hline \multicolumn{6}{|l|}{ CARGA DE TRABAJO } \\
\hline 1. Esta asignatura requiere que trabaje muy duro & 6.50 & 7.00 & 1.753 & 7.1 & 32.1 \\
\hline $\begin{array}{l}\text { 2. Parece que nunca tengo tiempo suficiente para hacer } \\
\text { el trabajo que requiere esta asignatura }\end{array}$ & 3.61 & 3.00 & 2.183 & 57.1 & 3.6 \\
\hline Media carga de trabajo & 5.05 & 5.00 & 1.46 & 10.7 & 0 \\
\hline
\end{tabular}

La tabla 4 presenta la satisfacción general con la asignatura, la cual alcanza una media de 8.68 , reflejando una valoración alta por parte del estudiantado. 
Tabla 4. Satisfacción general con la asignatura $(\mathrm{N}=28)$

\begin{tabular}{|l|c|c|c|c|c|}
\hline & Media & Mediana & $\begin{array}{c}\text { Desv. } \\
\text { típ. }\end{array}$ & $\begin{array}{c}\text { \% punt. } \\
\mathbf{1 - 3}\end{array}$ & $\begin{array}{c}\mathbf{\%} \text { punt. } \\
\mathbf{8 - 1 0}\end{array}$ \\
\hline Satisfacción general con la asignatura & 8.68 & 9 & 1.15 & 0 & 82.1 \\
\hline
\end{tabular}

Finalmente, la tabla 5 presenta las valoraciones medias de la variable aprendizaje significativo, apoyo de la profesora, apoyo de los compañeros, carga de trabajo y la satisfacción general con la asignatura, así como las correlaciones entre estas variables.

Tabla 5. Descriptivos y correlaciones $(\mathrm{N}=\mathbf{2 8})$

\begin{tabular}{lrrrcccc}
\hline & Media & $\begin{array}{c}\text { Desv. } \\
\text { típ. }\end{array}$ & 1 & 2 & 3 & 4 & 5 \\
\hline 1.Aprendizaje & 8.38 & 1.01 & 1 & & & & \\
significativo & 9.47 & 0.73 & $0.531^{* *}$ & 1 & & & \\
2.Apoyo profesora & 8.23 & 1.63 & 0.291 & 0.249 & 1 & & \\
3.Apoyo compañeros & 5.05 & 1.46 & -0.351 & -0.352 & -0.318 & 1 & \\
4.Carga trabajo & 8.68 & 1.15 & $0.742^{* *}$ & $0.703^{* *}$ & $0.423^{*}$ & -0.371 & 1 \\
5.Satisfacción & & & & & & & \\
\hline$* \mathrm{p}<0.05 ; * * \mathrm{p}<0.01$ & & & & & &
\end{tabular}

Como se observa, el nivel de satisfacción con la asignatura es elevado, y está relacionado significativamente con el aprendizaje significativo y el apoyo recibido por parte de la profesora y los compañeros. También se aprecia cómo la percepción de haber obtenido un aprendizaje significativo está relacionado positivamente con un mayor apoyo por parte de la profesora. Finalmente, se observa que no hay diferencias significativas en las variables analizadas en función de la asistencia o no a clase de una forma regular, evidenciándose que un $60 \%$ de los estudiantes asisten regularmente a clase (a más del $80 \%$ de las sesiones).

\section{Conclusiones}

Los resultados de la experiencia docente presentada nos permiten responder afirmativamente acerca de la contribución de la flipped classroom como metodología para alcanzar un aprendizaje significativo. Asimismo, este aprendizaje se consigue con unos niveles altos de satisfacción por parte del estudiantado. Estos resultados siguen la línea de trabajos previos que señalan la buena percepción que los estudiantes muestran con esta metodología (e.g. Asef-Vaziri, 2015; Prashar, 2015; Perdomo, 2016) y que se puede entender vinculada con un aprendizaje significativo. Además, los estudiantes no han percibido una alta carga de trabajo a pesar de ser una metodología activa en la que se requiere una mayor participación e implicación del estudiante en el proceso de enseñanzaaprendizaje. 
La satisfacción general con la asignatura se considera elevada y relacionada con el aprendizaje significativo y con el apoyo de la profesora. Este resultado apunta a la importancia que tiene la labor docente tanto de planificación como de implementación de la metodología, para lograr que los estudiantes sientan que el profesor les acompaña activamente durante su proceso de aprendizaje. En este sentido, Marqués (2016) señala que dar la vuelta a la clase no es sencillo y requiere de una mayor planificación de las sesiones, facilitando a los estudiantes los materiales que han de utilizar para exponerse a los contenidos así como las actividades de aprendizaje adecuadas para hacer dentro y fuera de clase. En el caso de esta asignatura el esfuerzo realizado por el docente es bien percibido por parte del estudiantado. Sin embargo, el cambio de metodología docente y la preparación que requiere la flipped classroom nos lleva a considerar la necesidad de replantear el cálculo de la carga docente del profesorado, que no debería reflejar sólo las horas de docencia presenciales y las tutorías, dado que el profesorado tiene que invertir más tiempo en la preparación de actividades y en la atención personalizada al estudiantado (AsefVaziri, 2015). También resulta un apoyo para el profesorado compartir experiencias docentes sobre la aplicación de esta metodología, de modo que se pueda contar con referentes y recomendaciones que hagan más efectiva la utilización de la flipped classroom.

\section{Agradecimientos}

Agradecemos el apoyo recibido desde la Unitat de Suport Educatiu (USE) dependiente del Vicerectorat d'Estudiants, Ocupació i Innovació Educativa de la Universitat Jaume I.

\section{Referencias}

Abeysekera, L. y Dawson, P. (2015). Motivation and cognitive load in the flipped classroom: definition, rationale and a call for research. Higher Education Research \& Development, 34(1), 114.

Asef-Vaziri, A. (2015). The flipped classroom of operations management: A not-for-cost-reduction platform. Decision Sciences Journal of Innovative Education, 13(1), 71-89.

Ausubel, D.P. (1982). Psicología Educativa: un punto de vista Cognoscitivo. México: Trillas.

Bain, K. (2006). El que fan els millors professors d'universitat. València: Publicacions de la Universitat de València.

Barreiro, M., y Novo, I. (2018). La enseñanza de Principios de Economía en el Grado en derecho: aula invertida como nueva metodología. En R. Casanova y C. Villó (Coords.), La motivación del estudiante universitario a través de la innovación docente (pp. 97-107). Barcelona: Huygens Editorial. 
Biggs, J., y Tang, C. (2011). Teaching for quality learning at university: what the student does (4th ed.). Berkshire: Society for Research into Higher Education \& Open University Press.

Cruz-Tomé, M.A. (2002). Seminario sobre Elaboración del Proyecto Docente. Universitat Jaume I, Castellón: Unitat de Suport Educatiu.

Dewey, J. (1916). Democracy and Education: an introduction to the philosophy of education. New York: Macmillan Company.

Esteban, R. (2018). Experiencia de aula invertida en la elaboración de materiales prácticos con alumnos de cuarto grado de derecho en la asignatura Derecho de la Seguridad Social. En R. Casanova y C. Villó (Coords.), La motivación del estudiante universitario a través de la innovación docente (pp. 81-95). Barcelona: Huygens Editorial.

Finkel, D. (2008). Dar clase con la boca cerrada. València: Publicacions de la Universitat de València.

Fulton, K. (2012). Upside down and inside out: Flip your classroom to improve student learning. Learning and Leading with Technology, 39(8), 12-17.

Guirao, J.A., Ferrer, E. y Olmedo, A. (2007). Escala para la medición de Aprendizaje Significativo en alumnos de Enfermería. Escola Infermeria. DOI: 10.13140/RG.2.1.4426.7040

Herreid, C. F., y Schiller, N. A. (2013). Case studies and the flipped classroom. Journal of College Science Teaching, 42(5), 62-66.

Lloret, T., y Mir, A. (2007). ¿Qué ha ocurrido en el primer año de implementación del EEES en algunas titulaciones?: Un primer balance en la UPF en términos de rendimiento académico, satisfacción y proceso de enseñanza-aprendizaje. Red U. Revista de Docencia Universitaria, 1, 117.

Marqués, M. (2016). Qué hay detrás de la clase al revés (flipped classroom). En Actas de las XXII JENUI. Universidad de Almería, 2016. p. 77-84.

Marqués, M., López, T., Ramón, V., Aliaga, J.I., Castaño, M.A., Catalán, S., Tortosa, V., Escrig, A.B., Flor, M.L., Vergara, M., y Balaguer, P. (2017). Adaptación de la Flipped Classroom para el aprendizaje de competencias en diversas titulaciones universitarias. VI Jornada Nacional sobre Estudios Universitarios y II Taller de Innovación Educativa. Universitat Jaume I, Castellón, noviembre 2017.

Perdomo, W. P. (2016). Estudio de evidencias de aprendizaje significativo en un aula bajo el modelo flipped classroom. Edutec. Revista Electrónica de Tecnología Educativa, (55), a325-a325.

Prashar, A. (2015). Assessing the flipped classroom in operations management: A pilot study. Journal of Education for Business, 90(3), 126-138.

Roehl, A., Reddy, S. L., y Shannon, G. J. (2013). The flipped classroom: An opportunity to engage millennial students through active learning. Journal of Family and Consumer Sciences, 105(2), 44-49.

Tyler, R.W. (1949). Basic Principles of Curriculum and Instruction. Chicago: University of Chicago Press. 
Van Wanrooy, B., Bewley, H., Bryson, A., Forth, J., Freeth, S., Stokes, L., y Wood, S. (2013), Employment relations in the shadow of recession: findings from the 2011 Workplace Employment Relations Study, Palgrave Macmillan.

Van Yperen, N.W., y Hagedoorn, M. (2003). Do high job demands increase intrinsic motivation or fatigue or both? The role of job control and job social support. Academy of Management Journal, 46(3), 339-348.

Vidal, C. (2012). El Espacio Europeo de Educación Superior y su Implantación en las Universidades Españolas. Revista Catalana de Dret Públic, 44, 253-283. 